\title{
INTEGRAL AVERAGING TECHNIQUES \\ FOR THE OSCILLATION \\ OF SECOND ORDER SUBLINEAR ORDINARY \\ DIFFERENTIAL EQUATIONS
}

\author{
CH. G. PHILOS
}

(Received 15 March 1983)

\begin{abstract}
New oscillation criteria are established for second order sublinear ordinary differential equations with alternating coefficients. These criteria are obtained by using an integral averaging technique and can be applied in some special cases in which other classical oscillation results are not applicable.
\end{abstract}

1980 Mathematics subject classification (Amer. Math. Soc.): 34 C 10, 34 C 15.

\section{Introduction}

The oscillation problem for second order nonlinear ordinary differential equations is of special importance. For some results concerning the problem we refer the reader to the paper by Wong [13] where a complete bibliography up to 1968 is given. Also, for a detailed account on second order nonlinear oscillation and its physical motivations we refer to a survey article by Ševelo [11]. In particular, for a survey on results for the so-called Emden-Fowler equation and a summary of some important historical developments concerning this equation, we refer to the article by Wong [16], where an extensive bibliography is contained. An interesting case is that of second order nonlinear ordinary differential equations with alternating coefficients. For such equations several oscillation criteria have been obtained. Some of the more important and useful tests involve the average

(c) 1986 Australian Mathematical Society $0263-6115 / 86 \$ A 2.00+0.00$ 
behavior of the integral of the alternating coefficient. These tests have been motivated by the classical averaging criterion of Wintner [12] (and its generalization by Hartman [2]) for the linear case. For such averaging techniques in the second order nonlinear oscillation, we choose to refer to the papers by Butler [1], Kamenev [3, 4], Kura [5], Kwong and Wong [6, 7], Onose [8], Philos [9, 10], Wong $[14,15]$, and to the references contained therein. The purpose of this paper is to proceed further in this direction for the case of sublinear ordinary differential equations of second order.

Throughout the paper, we restrict our attention only to solutions of the differential equations considered which exist on some ray $[T, \infty)$. A continuous real-valued function defined on ray $[T, \infty)$ is said to be oscillatory if it has arbitrarily large zeros. Otherwise, it is called nonoscillatory. A differential equation is called oscillatory if all its solutions are oscillatory.

Consider the second order nonlinear ordinary differential equation

$$
x^{\prime \prime}(t)+a(t) f[x(t)]=0,
$$

where

( $\alpha)$ the function $a$ is continuous on the interval $\left[t_{0}, \infty\right), t_{0}>0$, without any restriction on its sign,

$(\beta)$ the function $f$ is continuous on the real line $\mathbb{R}$ and has the sign property $y f(y)>0$ for all $y \neq 0$,

$(\gamma) f$ is continuously differentiable on $\mathbb{R}-\{0\}$ and satisfies $f^{\prime}(y)>0$ for $y \neq 0$,

$(\delta) f$ is strongly sublinear in the sense that

$$
\int_{0+} \frac{d y}{f(y)}<\infty \quad \text { and } \quad \int_{0-} \frac{d y}{f(y)}<\infty .
$$

For our purposes, we define

$$
F(y)=\int_{0+}^{y} \frac{d z}{f(z)} \quad \text { for } y>0 \text { and } \quad F(y)=\int_{0-}^{y} \frac{d z}{f(z)} \quad \text { for } y<0 .
$$

We also consider the constant $I_{f}$ defined by

$$
I_{f}=\min \left\{\frac{\inf _{y>0} F(y) f^{\prime}(y)}{1+\inf _{y>0} F(y) f^{\prime}(y)}, \frac{\inf _{y<0} F(y) f^{\prime}(y)}{1+\inf _{y<0} F(y) f^{\prime}(y)}\right\} \quad\left(0 \leqslant I_{f}<1\right) .
$$

Throughout the sequel, we suppose that $I_{f}>0$.

The special case where $f(y)=|y|^{\gamma}$ sgn $y, y \in \mathbb{R}(0<\gamma<1)$, i.e. the case of the differential equation

$$
x^{\prime \prime}(t)+a(t)|x(t)|^{\gamma} \operatorname{sgn} x(t)=0 \quad(0<\gamma<1),
$$

is of particular interest. In this case we can easily see that $I_{f}=\gamma$. 
Recently, Kura [5] and Kwong and Wong [7] presented two new oscillation criteria for the differential equation ( $\tilde{\mathrm{E}})$ which can be applied to the case $a(t)=t^{\lambda} \sin t$, where $t \geqslant t_{0}$ and $-\gamma<\lambda \leqslant 1-\gamma$, in which case other known oscillation tests including the criterion of Kamenev [3] are not applicable. In this paper, we extend (and improve) these criteria to the general case of the differential equation (E). We also deal with the oscillation in the more general case where a damped term is included, and with an asymptotic property in the forced case.

\section{Second order sublinear oscillation}

In this section we shall give two oscillation criteria for the differential equation (E).

THEOREM 1. Let $\varphi$ be a positive and twice continuously differential function on the interval $\left[t_{0}, \infty\right)$, and let $m$ be a continuous function on $\left[t_{0}, \infty\right)$ so that

$$
\limsup _{T \rightarrow \infty} \frac{1}{T} \int_{t}^{T} \int_{t}^{s}[\varphi(\tau)]^{I_{f}} a(\tau) d \tau d s \geqslant m(t) \quad \text { for every } t \geqslant t_{0} .
$$

Then equation $(\mathrm{E})$ is oscillatory if

$$
\int^{\infty} \frac{\left[m_{+}(t)\right]^{2}}{t} d t=\infty
$$

where $m_{+}(t)=\max \{m(t), 0\}, t \geqslant t_{0}$, and if, for some positive constant $c$,

$$
\left(\varphi^{\prime}\right)^{2} \leqslant c \varphi\left(-\varphi^{\prime \prime}\right) \quad \text { on }\left[t_{0}, \infty\right) .
$$

Proof. Let $x$ be a nonoscillatory solution on an interval $\left[T_{0}, \infty\right), T_{0} \geqslant t_{0}$, of the differential equation (E). Without loss of generality, we assume that $x(t) \neq 0$ for all $t \geqslant T_{0}$. Furthermore, we define

$$
w(t)=[\varphi(t)]^{I_{f}} F[x(t)], \quad t \geqslant T_{0} .
$$

Then for every $t \geqslant T_{0}$, we have

$$
\begin{aligned}
w^{\prime}(t) & =I_{f}[\varphi(t)]^{I_{f}-1} \varphi^{\prime}(t) F[x(t)]+[\varphi(t)]^{I_{f}} \frac{x^{\prime}(t)}{f[x(t)]} \\
& =I_{f} \frac{\varphi^{\prime}(t)}{\varphi(t)} w(t)+[\varphi(t)]^{I_{f}} \frac{x^{\prime}(t)}{f[x(t)]} .
\end{aligned}
$$


Therefore, using the product rule of differentiation and the method of completing the square, we obtain, for $t \geqslant T_{0}$ :

$$
\begin{aligned}
w^{\prime \prime}(t)= & {[\varphi(t)]^{I_{f}} \frac{x^{\prime \prime}(t)}{f[x(t)]}+I_{f}\left\{\frac{\varphi^{\prime \prime}(t)}{\varphi(t)}-\left[\frac{\varphi^{\prime}(t)}{\varphi(t)}\right]^{2}\right\} w(t)+I_{f} \frac{\varphi^{\prime}(t)}{\varphi(t)} w^{\prime}(t) } \\
& +I_{f} \frac{\varphi^{\prime}(t)}{\varphi(t)}\left[w^{\prime}(t)-I_{f} \frac{\varphi^{\prime}(t)}{\varphi(t)} w(t)\right] \\
& -\frac{1}{w(t)}\left[w^{\prime}(t)-I_{f} \frac{\varphi^{\prime}(t)}{\varphi(t)} w(t)\right]^{2} F[x(t)] f^{\prime}[x(t)] \\
\leqslant & {[\varphi(t)]^{I_{f}} \frac{x^{\prime \prime}(t)}{f[x(t)]}+I_{f} \frac{\varphi^{\prime \prime}(t)}{\varphi(t)} w(t) } \\
& -\frac{I_{f}}{1-I_{f}} \frac{1}{w(t)}\left[w^{\prime}(t)-\frac{\varphi^{\prime}(t)}{\varphi(t)} w(t)\right]^{2} .
\end{aligned}
$$

Upon integrating the above inequality twice and then multiplying by $-1 / T$, we obtain, for $T \geqslant t \geqslant T_{0}$, that

(1)

$$
\begin{aligned}
-\frac{w(T)}{T}+ & \frac{w(t)}{T}+\left(1-\frac{t}{T}\right) w^{\prime}(t) \geqslant \frac{1}{T} \int_{t}^{T} \int_{t}^{s}[\varphi(\tau)]^{I_{f}}\left\{-\frac{x^{\prime \prime}(\tau)}{f[x(\tau)]}\right\} d \tau d s \\
& +I_{f} \frac{1}{T} \int_{t}^{T} \int_{t}^{s} \frac{-\varphi^{\prime \prime}(\tau)}{\varphi(\tau)} w(\tau) d \tau d s \\
& +\frac{I_{f}}{1-I_{f}} \frac{1}{T} \int_{t}^{T} \int_{t}^{s} \frac{1}{w(\tau)}\left[w^{\prime}(\tau)-\frac{\varphi^{\prime}(\tau)}{\varphi(\tau)} w(\tau)\right]^{2} d \tau d s
\end{aligned}
$$

Now, by (i) and (iii), (1) gives

$$
\begin{aligned}
w^{\prime}(t) \geqslant & \limsup _{T \rightarrow \infty} \frac{1}{T} \int_{t}^{T} \int_{t}^{s}[\varphi(\tau)]^{I_{f}} a(\tau) d \tau d s \\
& +\liminf _{T \rightarrow \infty} \frac{w(T)}{T}+I_{f} \int_{t}^{\infty} \frac{-\varphi^{\prime \prime}(\tau)}{\varphi(\tau)} w(\tau) d \tau \\
& +\frac{I_{f}}{1-I_{f}} \int_{t}^{\infty} \frac{1}{w(\tau)}\left[w^{\prime}(\tau)-\frac{\varphi^{\prime}(\tau)}{\varphi(\tau)} w(\tau)\right]^{2} d \tau \\
\geqslant & m(t)+\liminf _{T \rightarrow \infty} \frac{w(T)}{T}+I_{f} \int_{t}^{\infty} \frac{-\varphi^{\prime \prime}(\tau)}{\varphi(\tau)} w(\tau) d \tau \\
& +\frac{I_{f}}{1-I_{f}} \int_{t}^{\infty} \frac{1}{w(\tau)}\left[w^{\prime}(\tau)-\frac{\varphi^{\prime}(\tau)}{\varphi(\tau)} w(\tau)\right]^{2} d \tau
\end{aligned}
$$


for all $t \geqslant T_{0}$, which proves that

$$
w^{\prime}(t) \geqslant m(t) \quad \text { for every } t \geqslant T_{0},
$$

$$
\begin{gathered}
\liminf _{T \rightarrow \infty} \frac{w(T)}{T}<\infty, \\
\int_{T_{0}}^{\infty} \frac{-\varphi^{\prime \prime}(\tau)}{\varphi(\tau)} w(\tau) d \tau<\infty,
\end{gathered}
$$

and

$$
\int_{T_{0}}^{\infty} \frac{1}{w(\tau)}\left[w^{\prime}(\tau)-\frac{\varphi^{\prime}(\tau)}{\varphi(\tau)} w(\tau)\right]^{2} d \tau<\infty
$$

Next, for $T \geqslant T_{0}$ we obtain

$$
\varphi(T)=\varphi\left(T_{0}\right)+\int_{T_{0}}^{T} \varphi^{\prime}(s) d s>\left(T-T_{0}\right) \varphi^{\prime}(T),
$$

which ensures that

$$
\limsup _{T \rightarrow \infty} \frac{T \varphi^{\prime}(T)}{\varphi(T)}<\infty .
$$

Also, by using (iii), we derive, for every $T \geqslant T_{0}$, that

$$
\begin{aligned}
& \int_{T_{0}}^{T} \frac{1}{w(\tau)}\left[w^{\prime}(\tau)-\frac{\varphi^{\prime}(\tau)}{\varphi(\tau)} w(\tau)\right]^{2} d \tau \\
&=\int_{T_{0}}^{T} \frac{\left[w^{\prime}(\tau)\right]^{2}}{w(\tau)} d \tau-2 \int_{T_{0}}^{T} \frac{\varphi^{\prime}(\tau)}{\varphi(\tau)} w^{\prime}(\tau) d \tau+\int_{T_{0}}^{T}\left[\frac{\varphi^{\prime}(\tau)}{\varphi(\tau)}\right]^{2} w(\tau) d \tau \\
&=\int_{T_{0}}^{T} \frac{\left[w^{\prime}(\tau)\right]^{2}}{w(\tau)} d \tau-2 \frac{\varphi^{\prime}(T)}{\varphi(T)} w(T)+2 \frac{\varphi^{\prime}\left(T_{0}\right)}{\varphi\left(T_{0}\right)} w\left(T_{0}\right) \\
&-2 \int_{T_{0}}^{T} \frac{-\varphi^{\prime \prime}(\tau)}{\varphi(\tau)} w(\tau) d \tau-\int_{T_{0}}^{T}\left[\frac{\varphi^{\prime}(\tau)}{\varphi(\tau)}\right]^{2} w(\tau) d \tau \\
& \geqslant \int_{T_{0}}^{T} \frac{\left[w^{\prime}(\tau)\right]^{2}}{w(\tau)} d \tau-2 \frac{T \varphi^{\prime}(T)}{\varphi(T)} \cdot \frac{w(T)}{T}+2 \frac{\varphi^{\prime}\left(T_{0}\right)}{\varphi\left(T_{0}\right)} w\left(T_{0}\right) \\
&-(c+2) \int_{T_{0}}^{T} \frac{-\varphi^{\prime \prime}(\tau)}{\varphi(\tau)} w(\tau) d \tau .
\end{aligned}
$$


Therefore,

$$
\begin{aligned}
\int_{T_{0}}^{\infty} & \frac{1}{w(\tau)}\left[w^{\prime}(\tau)-\frac{\varphi^{\prime}(\tau)}{\varphi(\tau)} w(\tau)\right]^{2} d \tau \\
& \geqslant \int_{T_{0}}^{\infty} \frac{\left[w^{\prime}(\tau)\right]^{2}}{w(\tau)} d \tau-2\left[\limsup _{T \rightarrow \infty} \frac{T \varphi^{\prime}(T)}{\varphi(T)}\right]\left[\liminf _{T \rightarrow \infty} \frac{w(T)}{T}\right] \\
& +2 \frac{\varphi^{\prime}\left(T_{0}\right)}{\varphi\left(T_{0}\right)} w\left(T_{0}\right)-(c+2) \int_{T_{0}}^{\infty} \frac{-\varphi^{\prime \prime}(\tau)}{\varphi(\tau)} w(\tau) d \tau
\end{aligned}
$$

which, because of (3), (4), (5) and (6), yields

$$
\int_{T_{0}}^{\infty} \frac{\left[w^{\prime}(\tau)\right]^{2}}{w(\tau)} d \tau<\infty
$$

Furthermore, by the Schwarz inequality, for $t \geqslant T_{0}$ we have

$$
\begin{aligned}
w(t) & =\left[\left[w\left(T_{0}\right)\right]^{1 / 2}+\left\{[w(t)]^{1 / 2}-\left[w\left(T_{0}\right)\right]^{1 / 2}\right\}\right]^{2} \\
& \leqslant 2 w\left(T_{0}\right)+2\left\{[w(t)]^{1 / 2}-\left[w\left(T_{0}\right)\right]^{1 / 2}\right\}^{2} \\
& =2 w\left(T_{0}\right)+\frac{1}{2}\left\{\int_{T_{0}}^{t} \frac{w^{\prime}(\tau)}{[w(\tau)]^{1 / 2}} d \tau\right\}^{2} \leqslant 2 w\left(T_{0}\right)+\frac{1}{2}\left(\int_{T_{0}}^{t} d s\right) \int_{T_{0}}^{t} \frac{\left[w^{\prime}(\tau)\right]^{2}}{w(\tau)} d \tau \\
& \leqslant 2 w\left(T_{0}\right)+\frac{1}{2} t \int_{T_{0}}^{\infty} \frac{\left[w^{\prime}(\tau)\right]^{2}}{w(\tau)} d \tau
\end{aligned}
$$

and consequently

$$
w(t) \leqslant t\left\{\frac{2 w\left(T_{0}\right)}{T_{0}}+\frac{1}{2} \int_{T_{0}}^{\infty} \frac{\left[w^{\prime}(\tau)\right]^{2}}{w(\tau)} d \tau\right\}, \quad t \geqslant T_{0} .
$$

Finally, by using (2), (7) and (8), we get

$$
\begin{aligned}
\int_{T_{0}}^{\infty} \frac{\left[m_{+}(t)\right]^{2}}{t} d t & \leqslant \int_{T_{0}}^{\infty} \frac{\left[w^{\prime}(t)\right]^{2}}{t} d t \\
& \leqslant\left\{\frac{2 w\left(T_{0}\right)}{T_{0}}+\frac{1}{2} \int_{T_{0}}^{\infty} \frac{\left[w^{\prime}(\tau)\right]^{2}}{w(\tau)} d \tau\right\} \int_{T_{0}}^{\infty} \frac{\left[w^{\prime}(t)\right]^{2}}{w(t)} d t<\infty,
\end{aligned}
$$

which contradicts (ii).

COROLlaRY 1. Let $\beta$ be a number with $0 \leqslant \beta<I_{f}$, and let $m$ be a continuous function on the interval $\left[t_{0}, \infty\right)$ so that

$$
\limsup _{T \rightarrow \infty} \frac{1}{T} \int_{t}^{T} \int_{t}^{s} \tau^{\beta} a(\tau) d \tau d s \geqslant m(t) \quad \text { for every } t \geqslant t_{0} .
$$

Then equation (E) is oscillatory if (ii) holds. 
Proof. The corollary follows immediately from Theorem 1 by choosing

$$
\varphi(t)=t^{\beta / I_{f}}, \quad t \geqslant t_{0} .
$$

REMARK 1. For the special case of the differential equation $(\tilde{\mathbf{E}})$, we have $I_{f}=\gamma$, and hence the oscillation criterion of Kura [5, Theorem 2] is a consequence of Corollary 1.

THEOREM 2. Let $\varphi$ be a positive and twice continuously differentiable function on the interal $\left[t_{0}, \infty\right)$ with

$$
\varphi^{\prime}>0 \text { and } \varphi^{\prime \prime} \leqslant 0 \quad \text { on }\left[t_{0}, \infty\right),
$$

and let $m$ be a continuous function on $\left[t_{0}, \infty\right)$ so that

(vi) $\quad \liminf _{T \rightarrow \infty} \frac{1}{T} \int_{t}^{T} \int_{t}^{s}[\varphi(\tau)]^{I_{f}} a(\tau) d \tau d s \geqslant m(t) \quad$ for every $t \geqslant t_{0}$.

Then equation (E) is oscillatory if

$$
\underset{t \rightarrow \infty}{\limsup }\left\{\int_{t_{0}}^{t}\left[\frac{\varphi^{\prime}(s)}{\varphi(s)}\right]^{2} s d s\right\}^{-1} \int_{t_{0}}^{t} \frac{\left[m_{+}(s)\right]^{2}}{s} d s=\infty
$$

Proof. Let $x$ be a solution on an interval $\left[T_{0}, \infty\right), T_{0}>t_{0}$, of the differential equation (E) with $x(t) \neq 0$ for all $t \geqslant T_{0}$, and let $w(t)=[\varphi(t)]^{I_{f} F}[x(t)], t \geqslant T_{0}$. Then, as in the proof of Theorem 1, we see that (1) holds for every $T, t$ with $T \geqslant t \geqslant T_{0}$. Thus, because of (v) and (vi), we have, for all $t \geqslant T_{0}$,

$$
\begin{aligned}
w^{\prime}(t) \geqslant & \liminf _{T \rightarrow \infty} \frac{1}{T} \int_{t}^{T} \int_{t}^{s}[\varphi(\tau)]^{I_{f}} a(\tau) d \tau d s \\
& +\limsup _{\tau \rightarrow \infty} \frac{w(T)}{T}+I_{f} \int_{t}^{\infty} \frac{-\varphi^{\prime \prime}(\tau)}{\varphi(\tau)} w(\tau) d \tau \\
& +\frac{I_{f}}{1-I_{f}} \int_{t}^{\infty} \frac{1}{w(\tau)}\left[w^{\prime}(\tau)-\frac{\varphi^{\prime}(\tau)}{\varphi(\tau)} w(\tau)\right]^{2} d \tau \\
\geqslant & m(t)+\limsup _{T \rightarrow \infty} \frac{w(T)}{T}+I_{f} \int_{t}^{\infty} \frac{-\varphi^{\prime \prime}(\tau)}{\varphi(\tau)} w(\tau) d \tau \\
& +\frac{I_{f}}{1-I_{f}} \int_{t}^{\infty} \frac{1}{w(\tau)}\left[w^{\prime}(\tau)-\frac{\varphi^{\prime}(\tau)}{\varphi(\tau)} w(\tau)\right]^{2} d \tau
\end{aligned}
$$

and

$$
\limsup _{T \rightarrow \infty} \frac{w(T)}{T}<\infty
$$


Also, as in the proof of Theorem 1, we arrive at (6). Furthermore, for every $T \geqslant T_{0}$, we obtain

$$
\begin{aligned}
\int_{T_{0}}^{T} \frac{\left[w^{\prime}(\tau)\right]^{2}}{w(\tau)} d \tau & =\int_{T_{0}}^{T} \frac{1}{w(\tau)}\left[w^{\prime}(\tau)-\frac{\varphi^{\prime}(\tau)}{\varphi(\tau)} w(\tau)\right]^{2} d \tau \\
& +2 \frac{\varphi^{\prime}(T)}{\varphi(T)} w(T)-2 \frac{\varphi^{\prime}\left(T_{0}\right)}{\varphi\left(T_{0}\right)} w\left(T_{0}\right) \\
& +2 \int_{T_{0}}^{T} \frac{-\varphi^{\prime \prime}(\tau)}{\varphi(\tau)} w(\tau) d \tau+\int_{T_{0}}^{T}\left[\frac{\varphi^{\prime}(\tau)}{\varphi(\tau)}\right]^{2} w(\tau) d \tau \\
\leqslant & \int_{T_{0}}^{T} \frac{1}{w(T)}\left[w^{\prime}(\tau)-\frac{\varphi^{\prime}(\tau)}{\varphi(\tau)} w(\tau)\right]^{2} d \tau \\
& +2 M \frac{T \varphi^{\prime}(T)}{\varphi(T)}+2 \int_{T_{0}}^{T} \frac{-\varphi^{\prime \prime}(\tau)}{\varphi(\tau)} w(\tau) d \tau+M \int_{T_{0}}^{T}\left[\frac{\varphi^{\prime}(\tau)}{\varphi(\tau)}\right]^{2} \tau d \tau
\end{aligned}
$$

where $M=\sup _{T \geqslant T_{0}} w(T) / T$ (and by (9), $M$ is finite). So, by taking (4), (5) and (6) into account, we conclude that there exists a positive constant $N$ such that

$$
\int_{T_{0}}^{t} \frac{\left[w^{\prime}(s)\right]^{2}}{w(s)} d s \leqslant N \int_{t_{0}}^{t}\left[\frac{\varphi^{\prime}(s)}{\varphi(s)}\right]^{2} s d s, \quad t \geqslant T_{0} .
$$

Finally, by (2) and (10), for $t \geqslant T_{0}$ we get

$$
\begin{aligned}
& \int_{t_{0}}^{t} \frac{\left[m_{+}(s)\right]^{2}}{s} d s=\int_{t_{0}}^{T_{0}} \frac{\left[m_{+}(s)\right]^{2}}{s} d s+\int_{T_{0}}^{t} \frac{\left[m_{+}(s)\right]^{2}}{s} d s \\
& \quad \leqslant \int_{t_{0}}^{T_{0}} \frac{\left[m_{+}(s)\right]^{2}}{s} d s+M \int_{T_{0}}^{t} \frac{\left[w^{\prime}(s)\right]^{2}}{w(s)} d s \\
& \quad \leqslant \int_{t_{0}}^{T_{0}} \frac{\left[m_{+}(s)\right]^{2}}{s} d s+M N \int_{t_{0}}^{t}\left[\frac{\varphi^{\prime}(s)}{\varphi(s)}\right]^{2} s d s \\
& \quad \leqslant\left\{\int_{t_{0}}^{t}\left[\frac{\varphi^{\prime}(s)}{\varphi(s)}\right]^{2} s d s\right\}\left[M N+\left\{\int_{t_{0}}^{T_{0}} \frac{\left[m_{+}(s)\right]^{2}}{s} d s\right\} /\left\{\int_{t_{0}}^{T_{0}}\left[\frac{\varphi^{\prime}(s)}{\varphi(s)}\right]^{2} s d s\right\}\right],
\end{aligned}
$$

which, by (vii), is a contradiction.

Remark 2. For the special case of the differential equation (Ẽ), Theorem 2 has recently been proved by Kwong and Wong [7, Theorem 1], under the additional assumption that

$$
\lim _{t \rightarrow \infty} \frac{1}{t} \int_{t_{0}}^{t} \int_{t_{0}}^{s}[\varphi(\tau)]^{\gamma} a(\tau) d \tau d s
$$

exists in $\mathbb{R}$. 
COROLLARY 2. Let $m$ be a continuous function on the interval $\left[t_{0}, \infty\right)$ such that

$$
\liminf _{T \rightarrow \infty} \frac{1}{T} \int_{t}^{T} \int_{t}^{s} \tau^{I^{\prime}} a(\tau) d \tau d s \geqslant m(t) \quad \text { for every } t \geqslant t_{0} .
$$

Then equation $(\mathrm{E})$ is oscillatory if

$$
\limsup _{t \rightarrow \infty} \frac{1}{\log t} \int_{t_{0}}^{t} \frac{\left[m_{+}(s)\right]^{2}}{s} d s=\infty
$$

ProOF. It suffices to apply Theorem 2 with $\varphi(t)=t, t \geqslant t_{0}$.

REMark 3. Provided that $\varphi$ is subject to (v), condition (vii) is satisfied if (ix) holds. Indeed, by using (v), we see that (6) is fulfilled, and hence there exists a positive constant $d$ such that $t \varphi^{\prime}(t) / \varphi(t) \leqslant d$ for all $t \geqslant t_{0}$. Thus, for all large $t$, we have

$$
\int_{t_{0}}^{t}\left[\frac{\varphi^{\prime}(s)}{\varphi(s)}\right]^{2} s d s \leqslant d^{2} \int_{t_{0}}^{t} \frac{d s}{s}=d^{2}\left(\log t-\log t_{0}\right) \leqslant 2 d^{2} \log t
$$

which proves our assertion.

REMARK 4. Condition (iii) of Theorem 1 is stronger than condition (v) required in Theorem 2. On the other hand, the other assumptions of Theorem 1 are weaker than the analogous ones in Theorem 2. In each of the cases (1), (2), (3) and (4) below, (iii) is satisfied, while in the cases (5) and (6), condition (iii) fails and (v) holds.

(1) $\varphi(t)=t^{\mu}, t \geqslant t_{0}$, where $0 \leqslant \mu<1$.

(2) $\varphi(t)=\log t, t \geqslant t_{0}$, where $t_{0}>1$.

(3) $\varphi(t)=t^{1 / 2} \log t, t \geqslant t_{0}$, where $t_{0}>1$.

(4) $\varphi(t)=\log ^{\mu} t, t \geqslant t_{0}$, where $\mu>0$ and $t_{0} \geqslant e^{\mu-1}$.

(5) $\varphi(t)=t+\log t, t \geqslant t_{0}$, where $t_{0}>1$.

(6) $\varphi(t)=t, t \geqslant t_{0}$.

REMARK 5. The case where $f(y)=|y|^{\gamma} \operatorname{sgn} y, y \in \mathbb{R}(0<\gamma<1)$ is the classical example of a function $f$ which satisfies all conditions imposed on it. Some more examples of such functions $f$ are the following ones.

(I) $f(y)=|y|^{\gamma} \operatorname{sgn} y+y, y \in \mathbb{R}(0<\gamma<1)$ with $\gamma / 2 \leqslant I_{f}<1$ (cf. [9]);

(II) $f(y)=|y|^{1 / 2} /\left(1+|y|^{1 / 4}\right)$ sgn $y, y \in \mathbb{R}$ with $I_{f}=1 / 4$;

(III) $f(y)=|y|^{\gamma}\{k+\sin [\log (1+|y|)]\} \operatorname{sgn} y, y \in \mathbb{R}(0<\gamma<1, k \geqslant 1+1 / \gamma)$ with $[(k-1) /(k+1)] \cdot[(\gamma k-\gamma-1) /(k+2 \gamma)] \leqslant I_{f}<1$ (cf. [9]). 
EXAMPLE 1. Consider the differential equation (E) with $a(t)=t^{\lambda} \sin t, t \geqslant t_{0}$, i.e. the equation

$$
x^{\prime \prime}(t)+t^{\lambda}(\sin t) f[x(t)]=0,
$$

where $\lambda$ is a real number. Moreover, consider the particular case of the differential equation

$$
x^{\prime \prime}(t)+t^{\lambda}(\sin t)|x(t)|^{\gamma} \operatorname{sgn} x(t)=0 \quad(0<\gamma<1) .
$$

Our purpose here is to prove that the differential equation $\left(\mathrm{E}_{\lambda}\right)$ is oscillatory for $\lambda>-I_{f}$. This result will be obtained from Theorem 1 in [9] when $\lambda>1-I_{f}$, and from Theorem 1 (or, more precisely, from Corollary 1) if $-I_{f}<\lambda \leqslant 1-I_{f}$. Theorem 1 in [9] ensures that $(E)$ is oscillatory if

$$
\limsup _{t \rightarrow \infty} \frac{1}{t} \int_{t_{0}}^{t} \int_{t_{0}}^{s} \tau^{\beta} a(\tau) d \tau d s=\infty \quad \text { for some } \beta \in\left[0, I_{f}\right]
$$

Note that $\left(\tilde{\mathrm{E}}_{\lambda}\right)$ is nonoscillatory if $\lambda<-\gamma$ (cf. Kwong and Wong [7]). Also, Butler [1] conjectures that $\left(\tilde{E}_{\lambda}\right)$ is oscillatory for $\lambda=-\gamma$, but this critical case remains open. Now, if $\delta$ is a real number, then for every $T, t$ with $T \geqslant t \geqslant t_{0}$, one can obtain

$$
\begin{aligned}
\frac{1}{T} \int_{t}^{T} \int_{t}^{s} \tau^{\delta} \sin & \tau d \tau d s \\
= & -T^{\delta-1} \sin T-2 \delta T^{\delta-2} \cos T+\delta(\delta-1)(\delta+1) T^{\delta-3} \sin T \\
& +\frac{1}{T}\left[t^{\delta} \sin t+2 \delta t^{\delta-1} \cos t-\delta(\delta-1)(\delta+1) t^{\delta-2} \sin t\right] \\
& -\delta(\delta-1)(\delta+1)(\delta-2) \frac{1}{T} \int_{t}^{T} s^{\delta-3} \sin s d s \\
& +\left(1-\frac{t}{T}\right)\left[t^{\delta} \cos t-\delta t^{\delta-1} \sin t-\delta(\delta-1) t^{\delta-2} \cos t\right] \\
& -\delta(\delta-1)(\delta-2) \int_{t}^{T} s^{\delta-3} \cos s d s
\end{aligned}
$$

If $\lambda>1-I_{f}$, then we put $\delta=I_{f}+\lambda>1$, and we get

$$
\underset{t \rightarrow \infty}{\limsup } \frac{1}{t} \int_{t_{0}}^{t} \int_{t_{0}}^{s} \tau^{I_{f}} a(\tau) d \tau d s=\limsup _{t \rightarrow \infty} \frac{1}{t} \int_{t_{0}}^{t} \int_{t_{0}}^{s} \tau^{\delta} \sin \tau d \tau d s=\infty,
$$

and consequently Theorem 1 in [9] ensures the oscillation of $\left(E_{\lambda}\right)$. Furthermore, we suppose that $-I_{f}<\lambda \leqslant 1-I_{f}$. If $-I_{f}<\lambda \leqslant 0$, we consider a number $\beta$ with $-I_{f}<-\beta<\lambda \leqslant 0$, while if $0<\lambda \leqslant 1-I_{f}$, we set $\beta=0$. Then $0 \leqslant \beta<I_{f}$. 
Moreover, we have $0<\delta \leqslant 1$, where $\delta=\beta+\lambda$. So, for all $t \geqslant t_{0}$, we obtain

$$
\begin{aligned}
\underset{T \rightarrow \infty}{\limsup } & \frac{1}{T} \int_{t}^{T} \int_{t}^{s} \tau^{\beta} a(\tau) d \tau d s=\limsup _{T \rightarrow \infty} \frac{1}{T} \int_{t}^{T} \int_{t}^{s} \tau^{\delta} \sin \tau d \tau d s \\
\geqslant & t^{\delta} \cos t-\delta t^{\delta-1} \sin t-\delta(\delta-1) t^{\delta-2} \cos t \\
& -\delta(\delta-1)(\delta-2) \int_{t}^{\infty} s^{\delta-3} \cos s d s \geqslant t^{\delta} \cos t-\mu
\end{aligned}
$$

where $\mu$ is positive constant. Namely, (iv) is satisfied with $m(t)=t^{\delta} \cos t-\mu$, $t \geqslant t_{0}$. Next, we consider an integer $N$ such that $2 N \pi-\pi / 4 \geqslant \max \left\{t_{0},(1+\right.$ $\left.\left.2^{1 / 2} \mu\right)^{1 / \delta}\right\}$. Then for all integers $n \geqslant N$, we have

$$
m(t) \geqslant 2^{-1 / 2} \quad \text { for every } t \in\left[2 n \pi-\frac{\pi}{4}, 2 n \pi+\frac{\pi}{4}\right] .
$$

Thus,

$$
\int_{t_{0}}^{\infty} \frac{\left[m_{+}(t)\right]^{2}}{t} d t \geqslant \frac{1}{2} \sum_{n=N}^{\infty} \int_{2 n \pi-\pi / 4}^{2 n \pi+\pi / 4} \frac{d t}{t}=\frac{1}{2} \sum_{n=N}^{\infty} \log \left(1+\frac{2}{8 n-1}\right)=\infty,
$$

i.e. (ii) is fulfilled. Hence, Corollary 1 can be applied to guarantee the oscillation of $\left(E_{\lambda}\right)$.

EXAMPLE 2. Consider the differential equation (Ẽ) with

$$
a(t)=t(t+\log t)^{-\gamma} \sin t, \quad t \geqslant t_{0}>1 .
$$

We define $\varphi(t)=t+\log t, t \geqslant t_{0}$, and we observe that (v) is fulfilled. Furthermore, for every $T, t$ with $T \geqslant t \geqslant t_{0}$, we obtain

$$
\begin{aligned}
\frac{1}{T} \int_{t}^{T} \int_{t}^{s}[\varphi(\tau)]^{\gamma} a(\tau) d \tau d s= & \frac{1}{T} \int_{t}^{T} \int_{t}^{s} \tau \sin \tau d \tau d s \\
= & -\sin T+\left(1-\frac{t}{T}\right)(t \cos t-\sin t) \\
& +\frac{1}{T}(-2 \cos T+t \sin t+2 \sin t),
\end{aligned}
$$

and consequently, for every $t \geqslant t_{0}$, we have

$$
\liminf _{T \rightarrow \infty} \frac{1}{T} \int_{t}^{T} \int_{t}^{s}[\varphi(\tau)]^{\gamma} a(\tau) d \tau d s=t \cos t-\sin t-1 \geqslant t \cos t-2 .
$$

Thus, (vi) holds with $m(t)=t \cos t-2, t \geqslant t_{0}$. We consider a number $t_{1}$ such that $t_{1} \geqslant \max \left\{t_{0}, 2^{5 / 2}\right\}$. Next, we choose an integer $N$ such that $2 N \pi-\pi / 4 \geqslant t_{1}$. Then, for every integer $n \geqslant N$, we have

$$
m(t) \geqslant 2^{-3 / 2} t \quad \text { for } t \in\left[2 n \pi-\frac{\pi}{4}, 2 n \pi+\frac{\pi}{4}\right] .
$$


Thus, for $n \geqslant N$, we get

$$
\int_{t_{0}}^{2 n \pi+\pi / 4} \frac{\left[m_{+}(s)\right]^{2}}{s} d s \geqslant \int_{2 n \pi-\pi / 4}^{2 n \pi+\pi / 4} \frac{\left[m_{+}(s)\right]^{2}}{s} d s \geqslant \frac{1}{8} \int_{2 n \pi-\pi / 4}^{2 n \pi+\pi / 4} s d s=\frac{\pi^{2} n}{8},
$$

and therefore

$$
\begin{aligned}
\limsup \frac{1}{\log t} \int_{t_{0}}^{t} \frac{\left[m_{+}(s)\right]^{2}}{s} d s & \geqslant \limsup _{n \rightarrow \infty} \frac{1}{\log (2 n \pi+\pi / 4)} \int_{t_{0}}^{2 n \pi+\pi / 4} \frac{\left[m_{+}(s)\right]^{2}}{s} d s \\
& \geqslant \lim _{n \rightarrow \infty} \frac{\pi^{2} n}{8 \log (2 n \pi+\pi / 4)}=\infty
\end{aligned}
$$

Hence, (ix) is satisfied and, consequently, (vii) holds, as noted in Remark 3. So, by Theorem 2, the differential equation under consideration is oscillatory.

\section{Oscillation in the damped case}

Theorems 1 and 2 can be extended to differential equations with damped term of the form

$$
x^{\prime \prime}(t)+q(t) x^{\prime}(t)+a(t) f[x(t)]=0,
$$

where $q$ is a nonnegative continuous function on the interval $\left[t_{0}, \infty\right)$. More precisely, we have the following more general theorems.

THEOREM 1'. Let $\varphi$ be a positive and twice continuously differentiable function on the interval $\left[t_{0}, \infty\right)$, and let $m$ be a continuous function on $\left[t_{0}, \infty\right)$ so that (i) holds. Suppose that (ii) holds and, for some positive constant $c$, that (iii) is satisfied. Moreover, suppose that

$$
\varphi^{I_{f}} q \text { is decreasing on }\left[t_{0}, \infty\right) \text {, }
$$

and that

$$
\int^{\infty} t[q(t)]^{2} d t<\infty
$$

Then the differential equation $\left(\mathrm{E}^{\prime}\right)$ is oscillatory.

Proof. Let $x$ be a solution on $\left[T_{0}, \infty\right), T_{0} \geqslant t_{0}$, of $\left(\mathrm{E}^{\prime}\right)$ with $x(t) \neq 0$ for all $t \geqslant T_{0}$, and let $w$ be defined as in the proof of Theorem 1 . Then, for every $T, t$ with $T \geqslant t \geqslant T_{0}$, (1) is satisfied. Furthermore, by taking into account (x), and by using the Bonnet theorem, we conclude that for any $s, t$ with $s \geqslant t \geqslant T_{0}$, there 
exists a number $\xi \in[t, s]$ such that

$$
\begin{aligned}
&-\int_{t}^{s}[\varphi(\tau)]^{I_{f}} q(\tau) \frac{x^{\prime}(\tau)}{f[x(\tau)]} d t=\int_{t}^{s}\left\{-[\varphi(\tau)]^{I_{f}} q(\tau)\right\} \frac{x^{\prime}(\tau)}{f[x(\tau)]} d t \\
& \quad=-[\varphi(\tau)]^{I_{f}} q(t) \int_{t}^{\xi} \frac{x^{\prime}(\tau)}{f[x(\tau)]} d t \\
&=[\varphi(\tau)]^{I_{f}} q(t) \int_{\xi}^{t} \frac{x^{\prime}(\tau)}{f[x(\tau)]} d t=[\varphi(\tau)]^{I_{f}} q(t) \int_{x(\xi)}^{x(t)} \frac{x^{\prime}(\tau)}{f[x(\tau)]} d t \\
&=[\varphi(\tau)]^{I_{f}} q(t) \int_{x(\xi)}^{x(t)} \frac{d y}{f(y)} \\
& \leqslant[\varphi(\tau)]^{I_{f}} q(t) F[x(t)]=q(t) w(t)
\end{aligned}
$$

since

$$
\int_{x(\xi)}^{x(t)} \frac{d y}{f(y)}< \begin{cases}0, & \text { if } x(\xi)>x(t) \\ \int_{+0}^{x(t)} \frac{d y}{f(y)}, & \text { if } x(\xi) \leqslant x(t)\end{cases}
$$

for $x>0$, and

$$
\int_{x(\xi)}^{x(t)} \frac{d y}{f(y)}< \begin{cases}0, & \text { if } x(\xi)<x(t) \\ \int_{-0}^{x(t)} \frac{d y}{f(y)}, & \text { if } x(\xi) \geqslant x(t)\end{cases}
$$

for $x<0$. Hence, for $T \geqslant t \geqslant T_{0}$, we have

$$
\begin{aligned}
-\frac{w(T)}{T}+\frac{w(t)}{T} & +\left(1-\frac{t}{T}\right)\left[w^{\prime}(t)+q(t) w(t)\right] \\
\geqslant & \frac{1}{T} \int_{t}^{T} \int_{t}^{s}[\varphi(\tau)]^{I_{f}} a(\tau) d \tau d s \\
& +I_{f} \frac{1}{T} \int_{t}^{T} \int_{t}^{s} \frac{-\varphi^{\prime \prime}(\tau)}{\varphi(\tau)} w(\tau) d \tau d s \\
& +\frac{I_{f}}{1-I_{f}} \frac{1}{T} \int_{t}^{T} \int_{t}^{s} \frac{1}{w(\tau)}\left[w^{\prime}(\tau)-\frac{\varphi^{\prime}(\tau)}{\varphi(\tau)} w(\tau)\right]^{2} d \tau d s
\end{aligned}
$$

Therefore, for every $t \geqslant T_{0}$, we obtain

$$
\begin{aligned}
w^{\prime}(t)+q(t) w(t) \geqslant & m(t)+\liminf _{T \rightarrow \infty} \frac{w(T)}{T}+I_{f} \int_{t}^{\infty} \frac{-\varphi^{\prime \prime}(\tau)}{\varphi(\tau)} w(\tau) d \tau \\
& +\frac{I_{f}}{1-I_{f}} \int_{t}^{\infty} \frac{1}{w(\tau)}\left[w^{\prime}(\tau)-\frac{\varphi^{\prime}(\tau)}{\varphi(\tau)} w(\tau)\right]^{2} d \tau
\end{aligned}
$$


Consequently, (3), (4) and (5) are satisfied, and we have

$$
w^{\prime}(t)+q(t) w(t) \geqslant m(t) \quad \text { for all } t \geqslant T_{0} .
$$

Next, as in the proof of Theorem 1, we derive (6), (7) and (8). So, by $(2)^{\prime},(7)$ and (8), we have

$$
\begin{aligned}
\int_{T_{0}}^{\infty} & \frac{\left[m_{+}(t)\right]^{2}}{t} d t \\
& \leqslant \int_{T_{0}}^{\infty} \frac{\left[w^{\prime}(t)+q(t) w(t)\right]^{2}}{t} d t \leqslant K \int_{T_{0}}^{\infty} \frac{\left[w^{\prime}(t)+q(t) w(t)\right]^{2}}{t} d t \\
& \leqslant 2 K \int_{T_{0}}^{\infty} \frac{\left[w^{\prime}(t)\right]^{2}}{w(t)} d t+2 K \int_{T_{0}}^{\infty}[q(t)]^{2} w(t) d t \\
& \leqslant 2 K \int_{T_{0}}^{\infty} \frac{\left[w^{\prime}(t)\right]^{2}}{w(t)} d t+2 K^{2} \int_{T_{0}}^{\infty} t[q(t)]^{2} d t
\end{aligned}
$$

where

$$
K=\frac{2 w\left(T_{0}\right)}{T_{0}}+\frac{1}{2} \int_{T_{0}}^{\infty} \frac{\left[w^{\prime}(\tau)\right]^{2}}{w(\tau)} d \tau<\infty .
$$

Thus, because of (ii) and (xi), we have arrived at a contradiction.

THEOREM 2 . Let $\varphi$ be a positive and twice continuously differentiable function on the interval $\left[t_{0}, \infty\right)$ satisfying $(v)$, and let $m$ be a continuous function on $\left[t_{0}, \infty\right)$ so that (vi) holds. Suppose that (vii) and (x) are satisfied, and that

$$
\underset{t \rightarrow \infty}{\limsup }\left\{\int_{t_{0}}^{t}\left[\frac{\varphi^{\prime}(s)}{\varphi(s)}\right]^{2} s d s\right\}^{-1} \int_{t_{0}}^{t} s[q(s)]^{2} d s<\infty .
$$

Then the differential equation $\left(\mathrm{E}^{\prime}\right)$ is oscillatory.

Proof. Let $x$ and $w$ be as in the proof of Theorem 1; and let $T_{0}>t_{0}$. Then (1)' is satisfied for $T \geqslant t \geqslant T_{0}$. Thus, for every $t \geqslant T_{0}$, we have

$$
\begin{aligned}
w^{\prime}(t)+ & q(t) w(t) \geqslant m(t)+\underset{T \rightarrow \infty}{\limsup } \frac{w(T)}{T} \\
& +I_{f} \int_{t}^{\infty} \frac{-\varphi^{\prime \prime}(\tau)}{\varphi(\tau)} w(\tau) d \tau+\frac{I_{f}}{1-I_{f}} \int_{t}^{\infty} \frac{1}{w(\tau)}\left[w^{\prime}(\tau)-\frac{\varphi^{\prime}(\tau)}{\varphi(\tau)} w(\tau)\right]^{2} d \tau,
\end{aligned}
$$

which ensures that (2)', (4), (5) and (9) hold. Furthermore, we can conclude that (10) is satisfied for some constant $N>0$. So, by taking (2)' and (10) into account, 
we obtain that, for every $t \geqslant T_{0}$,

$$
\begin{aligned}
\int_{t_{0}}^{t} \frac{\left[m_{+}(s)\right]^{2}}{s} & d s=\int_{t_{0}}^{T_{0}} \frac{\left[m_{+}(s)\right]^{2}}{s} d s+\int_{T_{0}}^{t} \frac{\left[m_{+}(s)\right]^{2}}{s} d s \\
& \leqslant \int_{t_{0}}^{T_{0}} \frac{\left[m_{+}(s)\right]^{2}}{s} d s+M \int_{T_{0}}^{t} \frac{\left[w^{\prime}(s)+q(s) w(s)\right]^{2}}{w(s)} d s \\
& \leqslant \int_{t_{0}}^{T_{0}} \frac{\left[m_{+}(s)\right]^{2}}{s} d s+2 M \int_{T_{0}}^{t} \frac{\left[w^{\prime}(s)\right]^{2}}{w(s)} d s+2 M \int_{T_{0}}^{t}[q(s)]^{2} w(s) d s \\
& \leqslant \int_{t_{0}}^{T_{0}} \frac{\left[m_{+}(s)\right]^{2}}{s} d s+2 M N \int_{t_{0}}^{t}\left[\frac{\varphi^{\prime}(s)}{\varphi(s)}\right]^{2} s d s+2 M^{2} \int_{T_{0}}^{t} s[q(s)]^{2} d s,
\end{aligned}
$$

where $M=\sup _{t \geqslant T_{0}} w(t) / t$ (and $M<\infty$ because of (9)). Hence, for all $t \geqslant T_{0}$, we have

$$
\begin{gathered}
\left\{\int_{t_{0}}^{t}\left[\frac{\varphi^{\prime}(s)}{\varphi(s)}\right]^{2} s d s\right\}^{-1} \int_{t_{0}}^{t} \frac{\left[m_{+}(s)\right]^{2}}{s} d s \leqslant 2 M^{2}\left\{\int_{t_{0}}^{t}\left[\frac{\varphi^{\prime}(s)}{\varphi(s)}\right]^{2} s d s\right\}^{-1} \int_{t_{0}}^{t} s[q(s)]^{2} d s \\
+2 M N+\left\{\int_{t_{0}}^{T_{0}} \frac{\left[m_{+}(s)\right]^{2}}{s} d s\right\} /\left\{\int_{t_{0}}^{T_{0}}\left[\frac{\varphi^{\prime}(s)}{\varphi(s)}\right]^{2} s d s\right\},
\end{gathered}
$$

which contradicts (vii) and (xii).

REMARK 6. By applying Theorem $1^{\prime}$ with $\varphi(t)=t^{\beta / I_{f}}, t \geqslant t_{0}\left(0 \leqslant \beta<I_{f}\right)$, we obtain the following result: let $\beta$ be a number with $0 \leqslant \beta<I_{f}$, and let $m$ be $a$ continuous function on $\left[t_{0}, \infty\right)$, so that (iv) holds. Then equation $\left(\mathrm{E}^{\prime}\right)$ is oscillatory if (ii) and (xi) are satisfied, and if $t^{\beta} q(t)$ is decreasing for $t \geqslant t_{0}$. Also, for $\varphi(t)=t$, $t \geqslant t_{0}$, Theorem $2^{\prime}$ leads to the next result: let $m$ be a continuous function on $\left[t_{0}, \infty\right)$ so that (viii) holds. Then equation $\left(\mathrm{E}^{\prime}\right)$ is oscillatory if (ix) is satisfied, if ${ }_{t}^{I_{f}} q(t)$ is decreasing for $t \geqslant t_{0}$, and if $\lim \sup _{t \rightarrow \infty}(1 / \log t) \int_{t_{0}}^{t} s[q(s)]^{2} d s<\infty$.

EXAMPLE 3. By applying Theorem $1^{\prime}$ with $\varphi(t)=t^{1 / 2}, t \geqslant t_{0}$, and with $m(t)=$ $t \cos t, t \geqslant t_{0}$, we conclude that the differential equation

is oscillatory.

$$
x^{\prime \prime}(t)+t^{-5 / 4} x^{\prime}(t)+t^{3 / 4} \sin t|x(t)|^{1 / 2} \operatorname{sgn} x(t)=0
$$

ExAmple 4. For $\varphi(t)=t, t \geqslant t_{0}$ and for $m(t)=t \cos t-2, t \geqslant t_{0}$, Theorem $2^{\prime}$ guarantees that the differential equation

$$
x^{\prime \prime}(t)+(1 / t) x^{\prime}(t)+t^{1 / 2} \sin t|x(t)|^{1 / 2} \operatorname{sgn} x(t)=0
$$

is oscillatory. 


\section{An asymptotic property in the forced case}

Now we shall give two results concerning the asymptotic behavior of the solutions of forced differential equations of the form

$$
x^{\prime \prime}(t)+a(t) f[x(t)]=b(t),
$$

where $b$ is a continuous function on the interval $\left[t_{0}, \infty\right)$.

Proposition 1. Let $\varphi$ be a positive and twice continuously differentiable function on the interval $\left[t_{0}, \infty\right)$, and let $m$ be a continuous function on $\left[t_{0}, \infty\right)$ so that (i) holds. Suppose that (ii) holds and, for some positive constant $c$, that (iii) is satisfied. Moreover, suppose that

$$
\int^{\infty} \frac{1}{t}\left\{\int_{t}^{\infty}[\varphi(s)]^{I_{f}}|b(s)| d s\right\}^{2} d t<\infty
$$

Then for all solutions $x$ of the differential equation $\left(\mathrm{E}^{*}\right)$, we have

$$
\liminf _{t \rightarrow \infty}|x(t)|=0
$$

Proof. Let $x$ be a solution of the differential equation $\left(\mathrm{E}^{*}\right)$ on an interval $\left[T_{0}, \infty\right), T_{0} \geqslant t_{0}$, with $\liminf _{t \rightarrow \infty}|x(t)|>0$. Obviously, $x$ is nonoscillatory. So we can suppose, without loss of generality, that $x(t) \neq 0$ for all $t \geqslant T_{0}$. Furthermore, let $w$ be defined as in the proof of Theorem 1 . Then (1) is satisfied for $T \geqslant t \geqslant T_{0}$. But, for any $T, t$ with $T \geqslant t \geqslant T_{0}$, we have

$$
\begin{aligned}
\frac{1}{T} \int_{t}^{T} \int_{t}^{s}[\varphi(\tau)]^{I_{f}} \frac{b(\tau)}{f[x(\tau)]} d \tau d s & \leqslant \frac{1}{T} \int_{t}^{T} \int_{t}^{s}[\varphi(\tau)]^{I_{f}} \frac{|b(\tau)|}{|f[x(\tau)]|} d \tau d s \\
& \leqslant \frac{1}{|f(\theta)|}\left(1-\frac{t}{T}\right) \int_{t}^{\infty}[\varphi(s)]^{I_{f}|b(s)| d s}
\end{aligned}
$$

where $\theta$ is a constant such that $x(t) \geqslant \theta>0$ for all $t \geqslant T_{0}$, or such that $x(t) \leqslant \theta<0$ for all $t \geqslant T_{0}$. Hence, for every $T, t$ with $T \geqslant t \geqslant t_{0}$, we have

$$
\begin{aligned}
(1)^{\prime \prime}-\frac{w(T)}{T}+\frac{w(t)}{T} & +\left(1-\frac{t}{T}\right)\left\{w^{\prime}(t)+\frac{1}{|f(\theta)|} \int_{t}^{\infty}[\varphi(s)]^{\left.I_{f}|b(s)| d s\right\}}\right. \\
\geqslant & \frac{1}{T} \int_{t}^{T} \int_{t}^{s}[\varphi(\tau)]^{I_{f}} a(\tau) d \tau d s \\
& +I_{f} \frac{1}{T} \int_{t}^{T} \int_{t}^{s} \frac{-\varphi^{\prime \prime}(\tau)}{\varphi(\tau)} w(\tau) d \tau d s \\
& +\frac{I_{f}}{1-I_{f}} \frac{1}{T} \int_{t}^{T} \int_{t}^{s} \frac{1}{w(\tau)}\left[w^{\prime}(\tau)-\frac{\varphi^{\prime}(\tau)}{\varphi(\tau)} w(\tau)\right]^{2} d \tau d s,
\end{aligned}
$$


and consequently, for $t \geqslant T_{0}$, we obtain

$$
\begin{aligned}
w^{\prime}(t)+\frac{1}{|f(\theta)|} \int_{t}^{\infty}[\varphi(s)]^{I^{\prime}}|b(s)| d s & \\
\geqslant & m(t)+\liminf _{T \rightarrow \infty} \frac{w(T)}{T}+I_{f} \int_{t}^{\infty} \frac{-\varphi^{\prime \prime}(\tau)}{\varphi(\tau)} w(\tau) d \tau \\
& +\frac{I_{f}}{1-I_{f}} \int_{t}^{\infty} \frac{1}{w(\tau)}\left[w^{\prime}(\tau)-\frac{\varphi^{\prime}(\tau)}{\varphi(\tau)} w(\tau)\right]^{2} d \tau
\end{aligned}
$$

Therefore, (3), (4) and (5) are fulfilled, and so

$(2)^{\prime \prime}$

$$
w^{\prime}(t)+\frac{1}{|f(\theta)|} \int_{t}^{\infty}[\varphi(s)]^{I_{f}}|b(s)| d s \geqslant m(t), \quad t \geqslant T_{0} .
$$

Furthermore, we may verify that (6), (7) and (8) hold. So, from (2)" (7), (8), and (xiii) we derive

$$
\begin{aligned}
\int_{T_{0}}^{\infty} \frac{\left[m_{+}(t)\right]^{2}}{t} d t \leqslant \int_{T_{0}}^{\infty} \frac{1}{t}\left\{w^{\prime}(t)+\frac{1}{|f(\theta)|} \int_{t}^{\infty}[\varphi(s)]^{I_{f}}|b(s)| d s\right\}^{2} d t \\
\leqslant 2 \int_{T_{0}}^{\infty} \frac{\left[w^{\prime}(t)\right]^{2}}{t} d t+\frac{2}{[f(\theta)]^{2}} \int_{T_{0}}^{\infty} \frac{1}{t}\left\{\int_{t}^{\infty}[\varphi(s)]^{I_{f}}|b(s)| d s\right\}^{2} d t \\
\leqslant 2\left\{\frac{2 w\left(T_{0}\right)}{T_{0}}+\frac{1}{2} \int_{T_{0}}^{\infty} \frac{\left[w^{\prime}(\tau)\right]^{2}}{w(\tau)} d \tau\right\} \int_{T_{0}}^{\infty} \frac{\left[w^{\prime}(t)\right]^{2}}{w(t)} d t \\
+\frac{2}{[f(\theta)]^{2}} \int_{T_{0}}^{\infty} \frac{1}{t}\left\{\int_{t}^{\infty}[\varphi(s)]^{I_{f}}|b(s)| d s\right\}^{2} d t<\infty
\end{aligned}
$$

which contradicts (ii).

Proposition 2. Let $\varphi$ be a positive and twice continuously differentiable function on the interval $\left[t_{0}, \infty\right)$ satisfying $(v)$, and let $m$ be a continuous function on $\left[t_{0}, \infty\right)$ so that (vi) holds. Suppose that (vii) is satisfied, and that

(xiv) $\limsup _{t \rightarrow \infty}\left\{\int_{t_{0}}^{t}\left[\frac{\varphi^{\prime}(s)}{\varphi(s)}\right]^{2} s d s\right\}^{-1} \int_{t_{0}}^{t} \frac{1}{s}\left\{\int_{s}^{\infty}[\varphi(\tau)]^{I_{f}}|b(\tau)| d \tau\right\}^{2} d s<\infty$.

Then for all solutions $x$ of the differential equation $\left(\mathrm{E}^{*}\right)$, we have

$$
\liminf _{t \rightarrow \infty}|x(t)|=0 \text {. }
$$

Proof. Let $x$ be a solution of $\left(\mathrm{E}^{*}\right)$ on $\left[T_{0}, \infty\right), T_{0}>t_{0}$, such that $x(t) \geqslant \theta>0$ for $t \geqslant T_{0}$, or such that $x(t) \leqslant \theta<0$ for $t \geqslant T_{0}$, where $\theta$ is a constant. Moreover, let $w$ be defined as in the proof of Theorem 1 . Then, for any $T, t$ with $T \geqslant t \geqslant T_{0}$, 
(1)" holds. So, for every $t \geqslant T_{0}$, we have

$$
\begin{aligned}
w^{\prime}(t) & +\frac{1}{|f(\theta)|} \int_{t}^{\infty}[\varphi(s)]^{I_{f}}|b(s)| d s \\
\geqslant & m(t)+\limsup _{T \rightarrow \infty} \frac{w(T)}{T}+I_{f} \int_{t}^{\infty} \frac{-\varphi^{\prime \prime}(\tau)}{\varphi(\tau)} w(\tau) d \tau \\
& +\frac{I_{f}}{1-I_{f}} \int_{t}^{\infty} \frac{1}{w(\tau)}\left[w^{\prime}(\tau)-\frac{\varphi^{\prime}(\tau)}{\varphi(\tau)} w(\tau)\right]^{2} d \tau
\end{aligned}
$$

and this implies that (2)", (4), (5) and (9) are satisfied. Furthermore, for some positive constant $N,(10)$ holds. So, for $t \geqslant T_{0}$, we obtain

$$
\begin{aligned}
\int_{t_{0}}^{t} \frac{\left[m_{+}(s)\right]^{2}}{s} d s \leqslant & \int_{t_{0}}^{T_{0}} \frac{\left[m_{+}(s)\right]^{2}}{s} d s \\
& +\int_{T_{0}}^{t} \frac{1}{s}\left\{w^{\prime}(s)+\frac{1}{|f(\theta)|} \int_{s}^{\infty}[\varphi(\tau)]^{I_{f}}|b(\tau)| d \tau\right\}^{2} d s \\
\leqslant & \int_{t_{0}}^{T_{0}} \frac{\left[m_{+}(s)\right]^{2}}{s} d s+2 \int_{T_{0}}^{t} \frac{\left[w^{\prime}(s)\right]^{2}}{s} d s \\
& +\frac{2}{[f(\theta)]^{2}} \int_{T_{0}}^{t} \frac{1}{s}\left\{\int_{s}^{\infty}[\varphi(\tau)]^{I_{f}}|b(\tau)| d \tau\right\}^{2} d s \\
\leqslant & \int_{t_{0}}^{T_{0}} \frac{\left[m_{+}(s)\right]^{2}}{s} d s+2 M N \int_{t_{0}}^{t}\left[\frac{\varphi^{\prime}(s)}{\varphi(s)}\right]^{2} s d s \\
& +\frac{2}{[f(\theta)]^{2}} \int_{t_{0}}^{t} \frac{1}{s}\left\{\int_{s}^{\infty}[\varphi(\tau)]^{I_{f}}|b(\tau)| d \tau\right\}^{2} d s
\end{aligned}
$$

where $M=\sup _{t \geqslant T_{0}} w(t) / t<\infty$. Thus, for every $t \geqslant T_{0}$, we have

$$
\begin{aligned}
\left(\int_{t_{0}}^{t}\left[\frac{\varphi^{\prime}(s)}{\varphi(s)}\right]^{2} s d s\right)^{-1} \int_{t_{0}}^{t} \frac{\left[m_{+}(s)\right]^{2}}{s} d s \\
\leqslant \frac{2}{[f(\theta)]^{2}}\left(\int_{t_{0}}^{t}\left[\frac{\varphi^{\prime}(s)}{\varphi(s)}\right]^{2} s d s\right)^{-1} \int_{t_{0}}^{t} \frac{1}{s}\left(\int_{s}^{\infty}[\varphi(\tau)]^{t_{f}}|b(\tau)| d \tau\right)^{2} d s \\
\quad+2 M N+\left(\int_{t_{0}}^{T_{0}} \frac{\left[m_{+}(s)\right]^{2}}{s} d s\right) /\left(\int_{t_{0}}^{T_{0}}\left[\frac{\varphi^{\prime}(s)}{\varphi(s)}\right]^{2} s d s\right)
\end{aligned}
$$

which contradicts (vii) and (xiv). 
Remark 7. From Propositions 1 and 2 we derive the following particular result: let $m$ be a continuous function on $\left[t_{0}, \infty\right)$. In each of the cases (I) and (II) below, all solutions $x$ of $\left(\mathrm{E}^{*}\right)$ satisfy $\liminf _{t \rightarrow \infty}|x(t)|=0$.

(I) (ii) and (iv) hold, and $\int^{\infty}(1 / t)\left[\int_{t}^{\infty} s^{\beta}|b(s)| d s\right]^{2} d t<\infty$, where $\beta$ is a number with $0 \leqslant \beta<I_{f}$.

(II) (viii) and (ix) hold, and $\int^{\infty} t^{I_{f}}|b(t)| d t<\infty$.

Remark 8. The methods used in proving Theorems 1', and $2^{\prime}$ and Propositions 1 and 2 can be applied in order to extend Propositions 1 and 2 to the more general case of forced differential equations with damped term of the form

$$
x^{\prime \prime}(t)+q(t) x^{\prime}(t)+a(t) f[x(t)]=b(t) .
$$

Example 5. By applying Proposition 1 with $\varphi(t)=t^{1 / 2}, t \geqslant t_{0}$, and with $m(t)=t \cos t, t \geqslant t_{0}$, we conclude that all solutions $x$ of the differential equation

$$
x^{\prime \prime}(t)+t^{3 / 4} \sin t|x(t)|^{1 / 2} \operatorname{sgn} x(t)=t^{-3 / 2}\left(\sin t+99 / 4 t^{5}\right)
$$

satisfy $\liminf \operatorname{in}_{t \rightarrow \infty}|x(t)|=0$. For example, $x(t)=t^{-9 / 2}, t \geqslant t_{0}$, is such a solution.

EXAMPLE 6. We choose $\varphi(t)=t, t \geqslant t_{0}$, and $m(t)=t \cos t-2, t \geqslant t_{0}$, and we apply Proposition 2 to the differential equation

$$
x^{\prime \prime}(t)+t^{1 / 2} \sin t|x(t)|^{1 / 2} \operatorname{sgn} x(t)=t^{-2}\left(\sin t+30 t^{-5}\right)
$$

to conclude that all solutions $x$ satisfy $\liminf _{t \rightarrow \infty}|x(t)|=0$. For example, $x(t)=$ $t^{-5}, t \geqslant t_{0}$, is such a solution.

REMARK 9. The results of this paper can be extended to more general differential equations involving the term $\left(r x^{\prime}\right)^{\prime}$ in place of the second derivative $x^{\prime \prime}$ of the unknown function $x$, where $r$ is a positive continuous function on $\left[t_{0}, \infty\right)$.

\section{References}

[1] G. J. Butler, "Integral averages and the oscillation of second order ordinary differential equations", SIAM J. Math. Anal. 11 (1980), 190-200.

[2] P. Hartman, "On nonoscillatory linear differential equations of second order", Amer. J. Math. 74 (1952), 389-400.

[3] I. V. Kamenev, "Some specifically nonlinear oscillation theorems", Mat. Zametki 10 (1971), 129-134 (Math. Notes 10 (1971), 502-505).

[4] I. V. Kamenev, "Oscillation criteria related to averaging of solutions of ordinary differential equations of second order", Differencial'nye Uravnenija 10 (1974), 246-252 (Differential Equations 10 (1974), 179-183). 
[5] T. Kura, "Oscillation theorems for a second order sublinear ordinary differential equation", Proc. Amer. Math. Soc. 84 (1982), 535-538.

[6] Man Kan Kwong and J. S. W. Wong, "On an oscillation theorem of Belohorec", SIAM J. Math. Anal. 14 (1983), 474-476.

[7] Man Kan Kwong and J. S. W. Wong, "On the oscillation and nonoscillation of second order sublinear equations", Proc. Amer. Math. Soc. 85 (1982), 547-551.

[8] H. Onose, "Oscillation criteria for second order nonlinear differential equations", Proc. Amer. Math. Soc. 51 (1975), 67-73.

[9] Ch. G. Philos, "Oscillation of sublinear differential equations of second order", Nonlinear A nal. 7 (1984), $1071-1080$.

[10] Ch. G. Philos, "A second order superlinear oscillation criterion", Canad. Math. Bull. 27 (1984), $102-112$.

[11] V. N. Ševelo, "Problems, methods and fundamental results in the theory of oscillation of solutions of nonlinear non-autonomous ordinary differential equations", pp. 142-157, Proceedings of the 2 nd All-Union Conference on Theoretical and Applied Mechanics, Moscow, 1965.

[12] A. Wintner, "A criterion of oscillatory stability", Quart. Appl. Math. 7 (1949), 115-117.

[13] J. S. W. Wong, "On the second order nonlinear oscillation", Funkcial. Ekvac. 11 (1968), $207-234$.

[14] J. S. W. Wong, "A second order nonlinear oscillation theorem", Proc. Amer. Math. Soc. 40 (1973), 487-491.

[15] J. S. W. Wong, "Oscillation theorems for second order nonlinear differential equations", Bull. Inst. Math. Acad. Sinica 3 (1975), 283-309.

[16] J. S. W. Wong, "On the generalized Emden-Fowler equation", SIAM Rev. 17 (1975), 339-360.

\section{Department of Mathematics \\ University of Ioannina \\ Ioannina}

\section{Greece}

